\title{
Molecular characterization of canine adenovirus type 2 in dogs from India
}

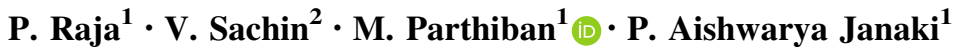

Received: 25 August 2020/Accepted: 17 April 2021/Published online: 5 May 2021

(C) Indian Virological Society 2021

\begin{abstract}
A total of 26 nasal swab samples were collected from dogs with gastroenteritis and respiratory tract infections in and around Chennai, India during 2019-20. All the samples were subjected to PCR using common primers for rapid diagnosis and differentiation of CAV1 and CAV2. Only one sample produced an amplicon of $1030 \mathrm{bp}$ indicating the presence of CAV2 which was confirmed by further sequencing. The analysis of the sequence revealed 100 per cent identity with other CAV type 2 isolates from Brazilian, Canadian and USA strains and 95.9 per cent identity with other Indian CAV2 strains. The phylogenetic analysis of E3 gene reveal two distinct clusters (Asian and America-Europe subgroup) in which our strain (ABT/ MVC/CAV2/001) grouped with CAV2 of America-Europe subgroup instead of Asian continent subgroup.This study confirms a novel CAV2 strain using molecular techniques which are genetically distinct in nature from other Indian CAV2 strains that is currently circulating in India.
\end{abstract}

Keywords Canine adenovirus $2 \cdot$ Molecular

characterization $\cdot$ PCR $\cdot$ Phylogenetic analysis $\cdot$ E3 gene

Canine adenovirus (CAV) is a linear non enveloped icosahedral double stranded DNA virus that belongs to the genus Mastadenovirus of the family Adenoviridae [1]. It causes severe fatal disease in canids and is endemic among

M. Parthiban

drparthiban66@gmail.com

1 Department of Animal Biotechnology, Faculty of Basic Sciences, Madras Veterinary College, Tamil Nadu Veterinary and Animal Sciences University, Chennai 600007, India

2 Hindustan Institute of Technology and Science, Chennai 603103, India domestic dogs. The CAV has two distinct serotypes CAV1 and CAV2. The CAV1 targets the digestive tract tissue and causative agent of infectious canine hepatitis (ICH) (also called Rubarth's disease), a life-threatening disease of puppies. The CAV2 replicates in respiratory epithelium and associated with respiratory diseases [2]. The disease also called as kennel cough and common among breeding stocks of dogs. The complete genome sequences of both CAV1 and CAV2 have been published by many workers $[3,4]$. However, studies on CAV2 are very limited. There is about $75 \%$ homology between CAV1 and CAV2 and the CAV2 genome consists of gene regions that encode various proteins with specific functions. The most important ones are E1A, E1B (19 K and $55 \mathrm{~K}), \mathrm{E} 3, \mathrm{E} 4, \mathrm{pIVa} 2$, pIIIa, pVI, pVII, pVIII, Pol, pTP, and DBP. E3 encodes the membrane proteins that modulate the host response to infection $[5,6]$. The open reading frame of the E3 regions of CAV1 and CAV2 encodes a $22 \mathrm{kDa}$ and a $40.7 \mathrm{kDa}$ polypeptide respectively and an additional region of about 510 nucleotides is present in CAV2. This region was considered to contribute to the observed biological differences between CAV1 and CAV2 [7].

Diagnosis and identification of CAV1 and CAV2 are usually based on virus isolation which is time consuming and laborious. Likewise, CAV1 and CAV2 can be difficult to differentiate in the laboratory by haemagglutination and neutralization test, especially when the infection occurs in the digestive tract [8]. In the recent years, for molecular detection and differentiation, a pair of common primers in the E3 and flanking regions for both CAV1 and CAV2 used in polymerase chain reaction, so as to provide a relatively simple method for diagnosing and differentiating the two serotypes of CAV [2]. In this study, nasal swab samples collected from dogs with gastroenteritis and respiratory illness were tested using PCR, targeting the E3 gene to 
obtain information about the occurrence of CAV2 in dogs and further this CAV2 strains were characterized using sequencing and phylogenetic analysis.

In this study, a total of 26 swab samples (14 nasal and 12 faecal swab samples) were collected from dogs with clinical signs of gastroenteritis and respiratory tract infection.The nasal swabs were resuspended in $1 \mathrm{ml}$ sterile phosphate buffer solution (1X PBS) and subjected to DNA extraction using QIAamp ${ }^{\circledR}$ virus isolation kits as per the manufacturer's instructions and subjected to PCR using one common pair of primers for detecting both CAV1 and CAV2. The primer sequences and thermal cyclic conditions already described by $\mathrm{Hu}$ et al. [2] were used in this study.The following primer sequences were used: Forward primer-HA1-5'-CGCGCTGAACATTACTACCTTGTC- ${ }^{\prime}$ and Reverse primer-HA2-5'-CCTAGAGCACTTCGTGTCCGCTT- $3^{\prime}$. The PCR amplification reactions were performed in a $25 \mu \mathrm{l}$ mixture containing $12.5 \mu \mathrm{l}$ of Pfu $2 \times$ DNA Polymerase Master Mix (GOLDBIO, USA), 20 pmol of each primers and $2 \mu \mathrm{l}$ of DNA template and the final volume was adjusted to $25 \mu \mathrm{l}$ by adding nuclease free water. The cycle condition for PCR amplification consists of initial denaturation $95{ }^{\circ} \mathrm{C}$ for $5 \mathrm{~min}$; 35 cycles of denaturation $96^{\circ} \mathrm{C}$ for $30 \mathrm{~s}$; annealing $58{ }^{\circ} \mathrm{C}$ for $1 \mathrm{~min}$; extension $72{ }^{\circ} \mathrm{C}$ for $1 \mathrm{~min}$ and final extension $72{ }^{\circ} \mathrm{C}$ for $5 \mathrm{~min}$. The final PCR amplified products were electrophoresed in $1.5 \%$ agarose gel and examined under gel documentation.

The PCR amplified products were purified using PCR gel purification kit (Bio Basic Inc, Canada) and sequencing was performed using both forward and reverse primers at M/s. Shrimpex Biotech, Chennai-600019 (India). The nucleotide sequence data was subjected to BLAST analysis (www.ncbi.nlm.nih.gov), assembled and analyzed using Seqman and MegAlign programs of Lasergene package (version 7.1.0) (DNA Star Inc. Madison, WI). Nucleotide sequence alignment was performed by ClustalW method with MegAlignTM program (DNA Star Inc), and the predicted amino acid sequence was analyzed by ProteanTM program of Lasergene (DNA Star Inc). Phylogenetic analysis of E3 gene, was performed using NeighborJoining method with 1000 bootstrap replication in the MEGAsoftware version 6.

The CAV2 is a highly contagious virus that is involved in canine respiratory tract disease, particularly in puppies that are kept in a crowded environment. The CAV2 is characterized by respiratory disorders, with clinical signs that include tonsillitis, pharyngitis, tracheitis, and bronchitis [12-14]. The CAV2 has an affinity for the epithelium of the upper airway, the nasal cavity, and especially the throat, larynx, and trachea tissue. This virus was first detected in dogs in a Canadian shelter in 1961 that had symptoms of upper respiratory tract infection [15]. In subsequent years, the presence of CAV-2 was reported in many countries such as Italy, Brazil, and India $[16,17,20]$.There have been several reports that CAV2 can also infect the intestinal tract, one of the major target organs for CAV- $1[9,10]$. However, there are reports that the recovered dogs from adenoviral infection and vaccinated dogs can shed virus continuously for prolonged period of time [11].

In the present study, the $\mathrm{E} 3$ gene region of $1030 \mathrm{bp}$ was amplified using the common pair of PCR primers and phylogenetic analysis was carried out to track the evolutionary changes at molecular level. Among 14 nasal and 12 faecal swab samples, only one nasal swab sample (3.84\%) found to be positive by PCR amplification and no amplification was observed in negative control and for CAV1 (Fig. 1). The results of the present study are in agreement with Timurkan et al. [18] who also reported that the dogs with respiratory system infection from two different shelters showed $2 \%$ and $2.8 \%$ CAV2 positivity, respectively. However, the results of the present study are in agreement with Schulz et al. [19] who also reported CAV2 in addition with other canine virus infections (canine parainfluenza virus, canine influenza virus and canine respiratory coronavirus) in dogs kept in crowded environment. Ramidi et al. [20], also screened a total of $302 \mathrm{fecal} / \mathrm{rectal}$ swabs collected from dogs with gastroenteritis in India and 4.9\% of samples found to be positive for CAV2 based on the E3 gene amplification. Similar findings were also reported by several workers all over the world regarding prevalence of CAV2 infections in dogs [13, 14]. However, contrary results have been reported by Parthiban et al. [8] who screened 32 numbers of nasal swabs samples initially by HA and confirmed presence of CAV1 from using PCR and sequencing of E3 gene in India.

The partial nucleotide sequences of the CAV2 E3 gene (912 bp) has been submitted to the Gen Bank with the accession number MT892837. The percentages of nucleic acid identity for the sequence of our CAV2 E3 genes was compared with CAV1 and CAV2 sequences available from NCBI and are shown in Table 1. The homology studies of ABT/MVC/CAV2/001 strain revealed 100 per cent identity with CAV2 of Canadian (U77082), Brazilian (KU315336, KU725673) and USA (AC_000020) strains. It also revealed 99.9 and 91.4 per cent identity with CAV2 strains of Italy (JX41684) and Chinese (GQ915311) respectively. While comparing with Indian CAV2 isolates and vaccine strain, the ABT/MVC/CAV2/001 strain revealed only 95.9 and 96.8 per cent identity (MN652563, MN652564, MN652565, MN652566, MN652567) respectively. While comparing with Indian CAV1 strains (EF090910 \& KT853097), the ABT/MVC/CAV2/001 strain revealed 42.1 and 22 per cent identity respectively. The results of the present study are in agreement with Timurkan et al. 
Fig.1 PCR amplification of E3 gene of Canine adenovirus. M$1 \mathrm{~Kb}$ Marker, $\mathrm{L}_{1^{-}}$Negative control, $\mathrm{L}_{2}$ - Positive control (vaccine), $\mathrm{L}_{3}-\mathrm{L}_{23}-\mathrm{CAV}$ suspected samples

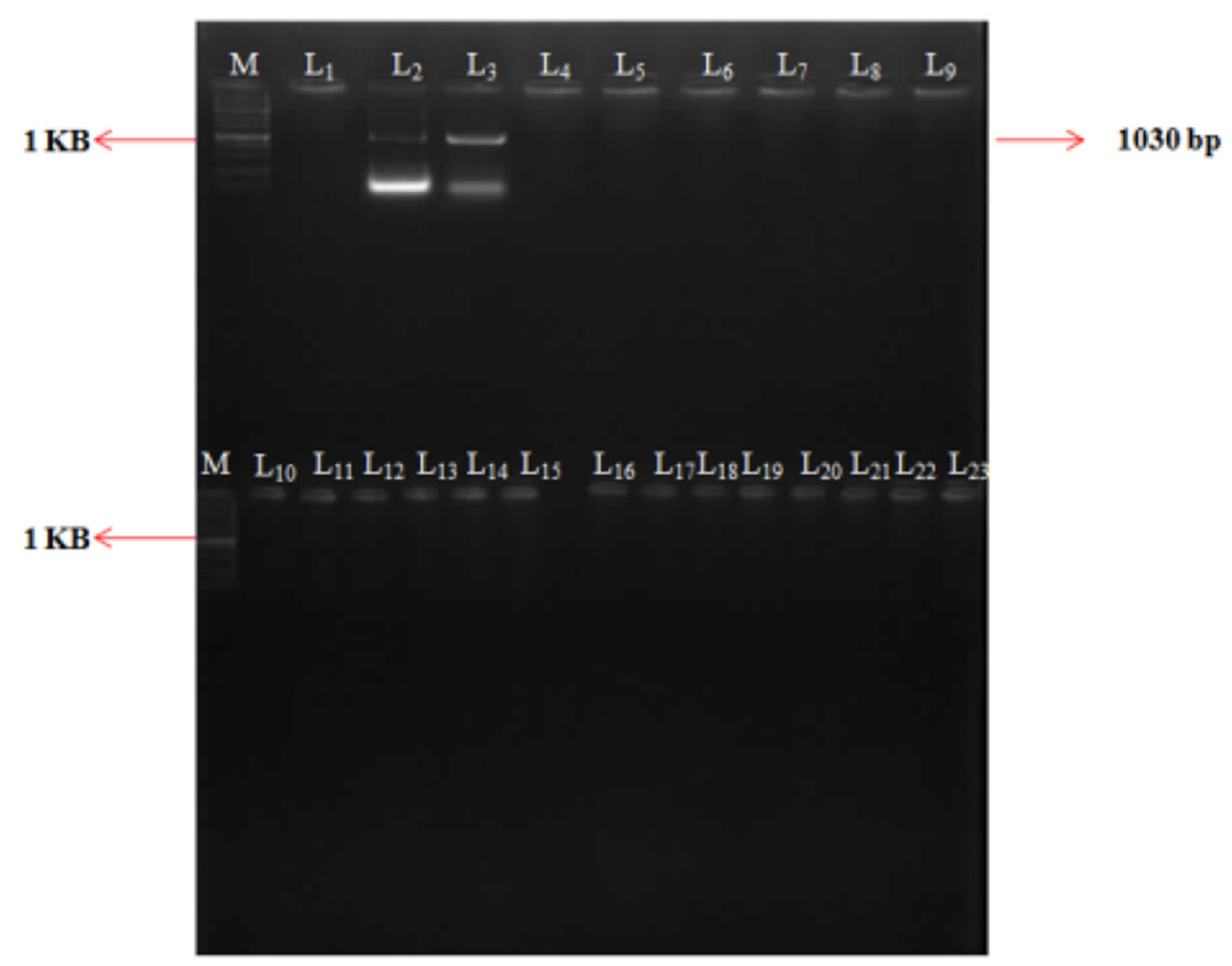

Table 1 Average nucleotide identity of canine adenovirus type 2 with the reference strain and with our strain

\begin{tabular}{|c|c|c|c|c|c|c|c|c|c|c|c|c|c|c|c|c|}
\hline \multicolumn{17}{|c|}{ Percent Identity } \\
\hline & 1 & 2 & 3 & 4 & 5 & 6 & 7 & 8 & 9 & 10 & 11 & 12 & 13 & 14 & 15 & \\
\hline 1 & & 50.4 & 97.7 & 99.9 & 84.5 & 100.0 & 100.0 & 100.0 & 98.8 & 98.8 & 98.8 & 98.8 & 100.0 & 100.0 & 99.9 & 1 \\
\hline 2 & 46.0 & & 15.1 & 38.1 & 89.1 & 11.9 & 38.3 & 32.6 & 22.4 & 22.4 & 22.4 & 22.4 & 51.0 & 42.5 & 14.2 & 2 \\
\hline 3 & 2.4 & 77.0 & & 88.9 & 8.9 & 97.6 & 89.4 & 91.9 & 94.8 & 94.8 & 94.8 & 94.8 & 97.7 & 91.4 & 97.5 & 3 \\
\hline 4 & 0.1 & 53.1 & 2.8 & & 20.7 & 99.9 & 99.9 & 97.2 & 93.4 & 93.4 & 93.4 & 93.4 & 99.9 & 99.9 & 94.3 & 4 \\
\hline 5 & 86.6 & 6.3 & 120.1 & 93.2 & & 8.2 & 20.9 & 18.1 & 13.0 & 13.0 & 13.0 & 13.0 & 84.1 & 22.3 & 8.6 & 5 \\
\hline 6 & 0.0 & 83.8 & 2.5 & 0.1 & 125.3 & & 100.0 & 100.0 & 99.0 & 99.0 & 99.0 & 99.0 & 100.0 & 100.0 & 100.0 & 6 \\
\hline 7 & 0.0 & 53.1 & 2.6 & 0.1 & 93.2 & 0.0 & & 97.7 & 93.9 & 93.9 & 93.9 & 93.9 & 100.0 & 100.0 & 94.8 & 7 \\
\hline 8 & 0.0 & 56.2 & 2.5 & 0.1 & 98.3 & 0.0 & 0.0 & & 96.3 & 96.3 & 96.3 & 96.3 & 100.0 & 99.5 & 97.3 & 8 \\
\hline 9 & 1.0 & 69.5 & 1.9 & 1.0 & 112.5 & 1.0 & 0.9 & 0.9 & & 100.0 & 100.0 & 100.0 & 98.8 & 95.9 & 99.0 & 9 \\
\hline 10 & 1.0 & 69.5 & 1.9 & 1.0 & 112.5 & 1.0 & 0.9 & 0.9 & 0.0 & & 100.0 & 100.0 & 98.8 & 95.9 & 99.0 & 10 \\
\hline 11 & 1.0 & 69.5 & 1.9 & 1.0 & 112.5 & 1.0 & 0.9 & 0.9 & 0.0 & 0.0 & & 100.0 & 98.8 & 95.9 & 99.0 & 11 \\
\hline 12 & 1.0 & 69.5 & 1.9 & 1.0 & 112.5 & 1.0 & 0.9 & 0.9 & 0.0 & 0.0 & 0.0 & & 98.8 & 95.9 & 99.0 & 12 \\
\hline 13 & 0.0 & 46.0 & 2.4 & 0.1 & 86.6 & 0.0 & 0.0 & 0.0 & 1.0 & 1.0 & 1.0 & 1.0 & & 100.0 & 99.9 & 13 \\
\hline 14 & 0.0 & 49.8 & 2.5 & 0.1 & 90.1 & 0.0 & 0.0 & 0.0 & 0.9 & 0.9 & 0.9 & 0.9 & 0.0 & & 96.8 & 14 \\
\hline 15 & 0.1 & 77.2 & 2.6 & 0.1 & 120.3 & 0.0 & 0.0 & 0.0 & 0.9 & 0.9 & 0.9 & 0.9 & 0.1 & 0.0 & & 15 \\
\hline & 1 & 2 & 3 & 4 & 5 & 6 & 7 & 8 & 9 & 10 & 11 & 12 & 13 & 14 & 15 & \\
\hline
\end{tabular}

\author{
AC_000020 CAV2 USA \\ EF090910 CAV1 \\ GQ915311 CAV2 CHINA \\ JX416842 CAV2 ITALY \\ KT853097 CAV1 \\ KU315336 CAV2 BRAZIL \\ KU725673 CAV2 BRAZIL \\ KU725674 CAV2 BRAZIL \\ MN652563 CAV2 INDIA \\ MN652564 CAV2 INDIA \\ MN652565 CAV2 INDIA \\ MNN652566 CAV2 INDIA \\ U77082 CAV2 CANADA \\ ABT MVC CAV2 001 \\ MN652567
}

[18] who also reported the similarities among the Turkish strains in about $97.7-98.9 \%$ and $84.2-99.7 \%$ nucleotide similarities with Chinese reference strains.

The phylogenetic analyses of the CAV2 based on E3 gene revealed a formation of two different clusters (subgroups) with other CAV2 strains (America-Europe and Asian continent subgroup) from Genbank, NCBI databases (Fig. 2). The ABT/MVC/CAV2/001 strain belongs to
CAV2 strain and grouping with Brazilian, Italy, Canadian and USA strains of CAV2 which in turn forms the America-Europe subgroup where previous study in India, showed the close relationship with Chinese and Turkish strains which in turn comprising the Asian continent subgroup. This present study revealed the unique nature of new strain that is currently circulating in India is genetically distinct novel strain from other Indian CAV2 strains. 


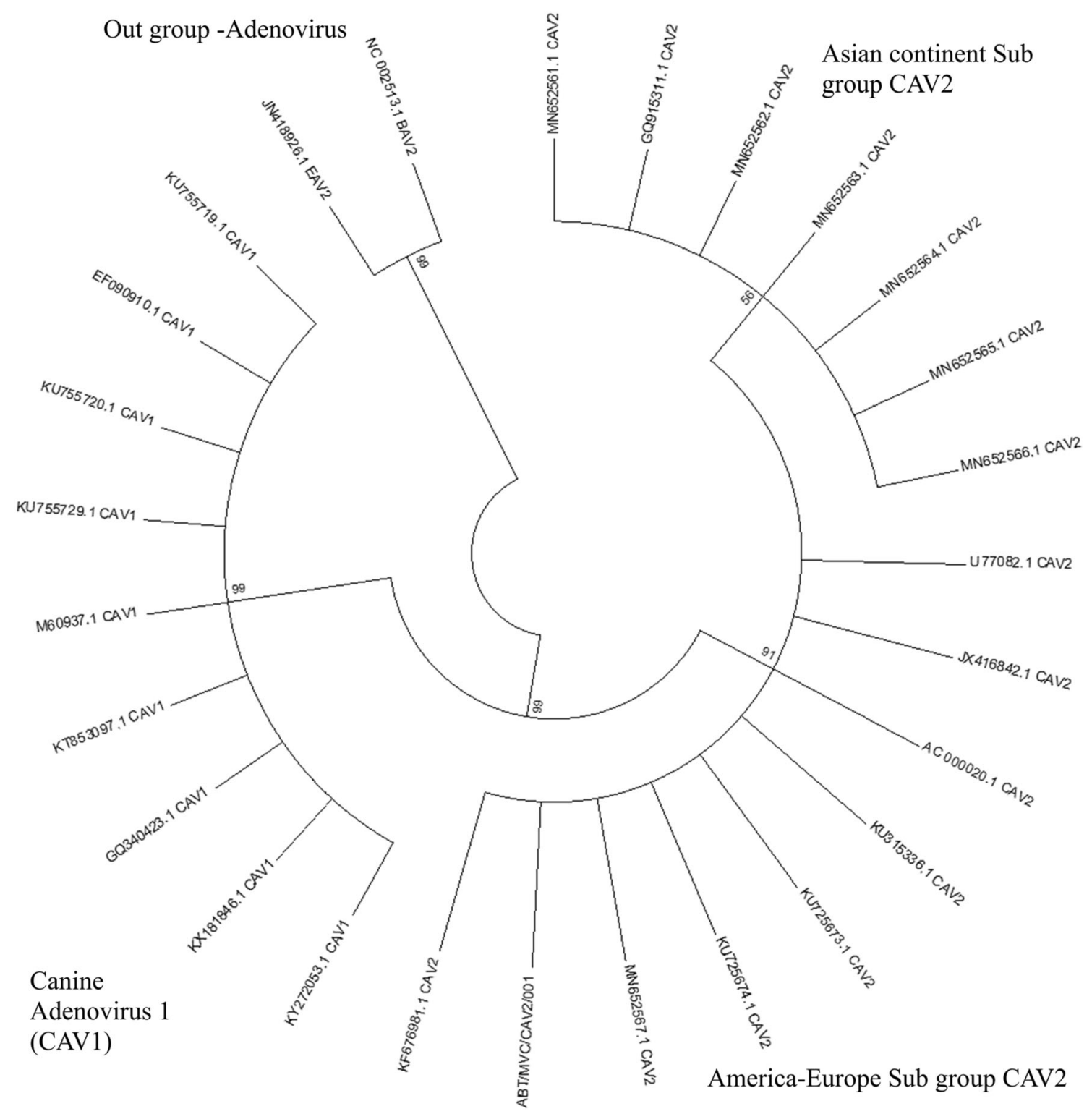

Fig.2 Phylogenetic Analysis of Canine Adenovirus type 2 based on E3 gene. Phylogenetic tree based on a 842 nucleotide sequence of 26 strains of adenoviruses (CAV-1, CAV-2, EAV1 and BAV2) generated

by using the neighbor- joining algorithm, test of phylogeny is the bootstrap method with 1000 replicates, using the MEGA 6.0 software program. Equine, and bovine adenoviruses were used as an out group

The results of the present study in agreement with Ramidi et al. [20], who also reported circulation of novel strain and phylogenetically classified as three groups instead of two groups with the novel group of CAV-2 strains in India.The CAV1 forms single cluster and Equine and Bovine adenovirus used as out group.

The partial 842 nucleotide sequence of $\mathrm{ABT} / \mathrm{MVC} /$ CAV2/001 of E3 gene coded for 254 amino acids were shown in Table 2. The deduced amino acid sequence of ABT/MVC/CAV2/001 is compared with other Indian (Protein ID: QJZ27972-QJZ27977), Chinese (Protein ID: ACV90490), Italy (Protein ID: ADN96228-ADN96229, AFZ61865), Brazilian (Protein ID: ANF99502) and

Canadian (Protein ID: AAB38732) isolates of CAV2.The deduced amino acid ABT/MVC/CAV2/001 revealed the consistent amino acid change at positions $126(\mathrm{~A} \rightarrow \mathrm{T})$, $136(\mathrm{M} \rightarrow \mathrm{K}), 138(\mathrm{~N} \rightarrow \mathrm{D}), 172(\mathrm{~T} \rightarrow \mathrm{V}), 186(\mathrm{E} \rightarrow \mathrm{D})$, $216(\mathrm{~V} \rightarrow \mathrm{M}), 222(\mathrm{~N} \rightarrow \mathrm{D}), 227(\mathrm{~V} \rightarrow \mathrm{I})$, and $254(\mathrm{~S} \rightarrow \mathrm{P})$ are predominant mutations in CAV-2 of Indian strain while comparing with Chinese strain (ACV90490) and more similar with Brazilian, Italy and USA strains of CAV2. While comparing with Indian CAV2 isolates, the ABT/ MVC/CAV2/001 revealed an additional amino acid changes at the position 251 to 255 as "KIRPM" instead of "ENQAH". This is characteristic unique amino acid mutations present in the newly reported Indian strain. The 
Table 2 Alignment of the deduced amino acid sequences of the partial-length E3 gene (253 amino acids) amplified using PCR

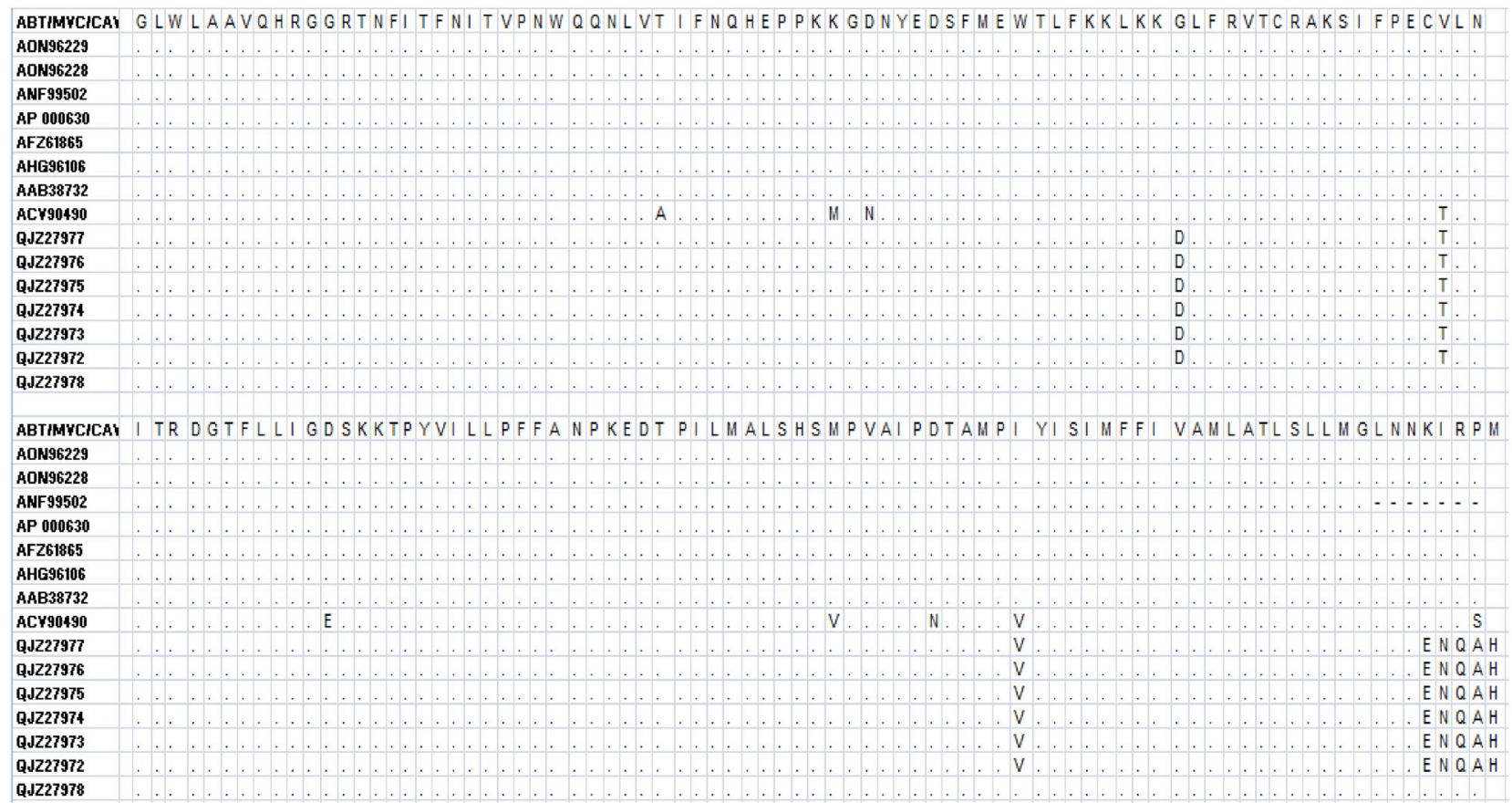

results of the present study are in agreement with Timurkan et al. [18] who also reported the fixed substitutions changes at amino acid position 126, 136, 138, 172, 186, 216, 222, 227 and 254 could be the result of the differences in the CAV-2 subgroups. There are nine different amino acids differences reported between the China-Indian strains. These amino acid differences suggest that the Indian strain belongs to America-Europe subgroup.

In the present study molecular characterization and evolutionary analyses based on the E3 gene of CAV2 revealed a novel strain that is currently circulating in field and also throw lights on needs of epidemiological surveillance of different subgroups of CAV type 2 in India. The CAV2 strain exhibited $96.8 \%$ and $100 \%$ homology at nucleotide and amino acid level respectively. Hence, the current vaccine strain may be used for control of canine adenovirus type 2 infections. However, more number of samples to be analysed to derive the conclusion about the relationship between vaccine and field strains.

\section{Declarations}

Conflict of interest The authors declare that they have no conflict of interest.

Human and animal rights This study was carried out under self financing scheme "Human Resource Development" of this Department of Animal Biotechnology.

\section{References}

1. Appel MJ. Canine adenovirus type 1 (infectious canine hepatitis virus). In: Horzinek MC.(ed.) Virus Infections of Vertebrates, Virus infections of Carnivores. Elsevier Science Publishers, Amsterdam, The Netherland. 1987;1:29-43.

2. Hu RL, Huang G, Qiu W, Zhong ZH, Xia XZ, Yin Z. Detection and differentiation of CAV-1 and CAV-2 by polymerase chain reaction. Vet Res Commun. 2001;25:777-84.

3. Morrison MD, Onions DE, Nicolson L. Complete DNA sequence of canine adenovirus type 1. J Gen Virol. 1997. https://doi.org/10. 1099/0022-1317-78-4-873.

4. Campbell JB, Zhao Y. Complete DNA sequence and genomic organization of canine adenovirus type 2. In Genbank (Direct submitted). 1996.

5. Burgert HG, Blusch JH. Immunomodulatory functions encoded by the E3 transcription unit of adenoviruses. Virus Genes. 2000. https://doi.org/10.1023/A:1008135928310.

6. Lichtenstein DL, Toth K, Doronin K, Tollefson AE, Wold WS. Functions and mechanisms of action of the adenovirus E3 proteins. Int Rev Immunol. 2004. https://doi.org/10.1080/ 08830180490265556.

7. Linne T. Differences in the E3 regions of the canine adenovirus type 1 and type 2. Virus Res. 1992;23:119-33.

8. Parthiban MK, Kumanan K, Sunder S, Kumar S, Kathiresan D. Molecular detection of canine adenovirus using polymerase chain reaction and sequencing. J Vet Animal Sci. 2009;5:140-2.

9. Hamelin C, Jouvenne P, Assaf R. Genotypic characterization of type-2 variants of canine adenovirus. Am $\mathrm{J}$ Vet Res. 1986;47:625-30.

10. Macartney L, Cavanagh HMA, Spibey N. Isolation of canine adenovirus- 2 from the faeces of dogs with enteric disease and its unambiguous typing by restriction endonuclease mapping. Res Vet Sci. 1988;44:9-14. 
11. Taguchi M, Namikawa K, Maruo T, Orito K, Lynch J, Sahara H. Antibody titers for canine parvovirus type-2, canine distemper virus, and canine adenovirus type- 1 in adult household dogs. Can Vet J. 2011;52:983-6.

12. Pratelli A, Martella V, Elia G, Tempesta M, Guarda F, Capucchio MT, Carmichael LE, Buonavoglia C. Severe enteric disease in an animal shelter associated with dual infections by canine adenovirus type 1 and canine coronavirus. $J$ Vet Med B. 2001;48:385-92.

13. Decaro N, Campolo M, Elia G, Buonavoglia D, Colaianni ML, Lorusso A, Mari V, Buonavoglia C. Infectious canine hepatitis: an "old" disease reemerging in Italy. Res Vet Sci. 2007;83:269-73.

14. Mochizuki M, Yachi A, Ohshima T, Ohuchi A, Ishida T. Etiologic study of upper respiratory infections of household dogs. J Vet Med Sci. 2008;70:563-9.

15. Ditchfield JL, Macpherson W, Zbitnew A. Association of a canine adenovirus (Toronto A 26/61) with an outbreak of laryngotracheitis ("kennel cough") a preliminary report. Can Vet J. 1968;3:238-47.

16. Balboni A, Verin R, Morandi F, Poli A, Prosperi S, Battilani M. Molecular epidemiology of canine adenovirus type 1 and type 2 in free-ranging red foxes (Vulpesvulpes) in Italy. Vet Microbiol. 2013. https://doi.org/10.1016/j.vetmic.2012.11.015.

17. Silva AP, Bodnar L, Headley SA, Alfieri AF, Alfieri AA. Molecular detection of canine distemper virus (CDV), canine adenovirus $\mathrm{A}$ type 1 and 2 (CAdV-1 and CAdV-2), and canine parvovirus type $2(\mathrm{CPV}-2)$ in the urine of naturally infected dogs. SeminCiêncAgrár. 2014;35:3231-6.

18. Timurkan MO, Aydin H, Alkan F. Detection and molecular characterization of canine adenovirus type 2 (CAV-2) in dogs with respiratory tract symptoms in shelters in Turkey. Vet Arch. 2018;88:467-79.

19. Schulz BS, Kurz S, Weber K, Balzer HJ, Hartmann K. Detection of respiratory viruses and Bordetellabronchiseptica in dogs with acute respiratory tract infections. Vet J. 2014;201:365-9.

20. Ramidi A, Ganji VK, Buddala B, Yella NR, Manthani GP, Putty K. E3 gene-based genetic characterization of canine adenovirus-2 isolated from cases of canine gastroenteritis in India revealed a novel group of the virus. Intervirology. 2019;62:216-21.

Publisher's Note Springer Nature remains neutral with regard to jurisdictional claims in published maps and institutional affiliations. 